\title{
Design and Characterization of a Miniaturized Epi-illuminated Microscope
}

\author{
Kartikeya Murari, Elliot Greenwald, Ralph Etienne-Cummings, Gert Cauwenberghs and Nitish Thakor
}

\begin{abstract}
The ability to observe functional and morphological changes in the brain is critical in understanding behavioral and developmental neuroscience. With advances in electronics and miniaturization, electrophysiological recordings from awake, behaving animals has allowed investigators to perform a multitude of behavioral studies by observing changes as an animal is engaged in certain tasks. Imaging offers advantages of observing structure as well as function, and the ability to monitor activity over large areas. However, imaging from an awake, behaving animal has not been explored well. We present the design and characterization of a miniaturized epiilluminated optical system that is part of a larger goal to perform optical imaging in awake, behaving animals. The system comprises of a tunable light source and imaging optics in a small footprint of $18 \mathrm{~mm}$ diameter, $18 \mathrm{~mm}$ height and weight 5.7 grams. It offers a spatial illumination non-uniformity of $3.2 \%$ over a maximum field of view of $1.5 \mathrm{~mm} \times 1.5 \mathrm{~mm}$, negligible temporal illumination and temperature variation and controllable magnification. Uncorrected radial distortion was $5.3 \%$ (corrected to $1.8 \%$ ) and the spatial frequency response was comparable to a reference system. The system was used to image cortical vasculature in an anesthetized rat.
\end{abstract}

\section{INTRODUCTION}

The vast majority of small animal imaging today is carried out on anesthetized animals that are restrained in a stereotaxic frame. Depending on the drug, anesthesia can suppress regulatory mechanisms and normal functioning of the brain [1], [2]. Restraining the animal also causes stress and limits the range of behavioral studies that can be performed. With advances in integrated circuit technology, electrophysiological recordings from freely moving animals have been performed [3], [4], making possible a wide variety of behavioral studies. However, imaging in a similar scenario has not been explored well. The advantages of imaging over electrophysiology include measurements from larger areas and the ability to visualize structure in addition to function.

Over the years, some efforts have been made to image from awake, behaving animals. Harper et al. [5] reported on imaging from a cat using a miniaturized CCD camera. Gmitro et al. developed a confocal microendoscope [6] for in vivo imaging. Miniaturized two-photon [7]-[9] and fluorescent [10], [11] microscopes attached to rodent skulls have been reported. Most of these systems rely on a bundle of optical fibers to transfer the image from the focal plane

This work was supported by the NIH grant R01AG029681

K. Murari, E. Greenwald and N. Thakor are with the department of Biomedical Engineering, Johns Hopkins School of Medicine, Baltimore, MD, USA. \{kartik, egreenw3, nitish\}ejhu.edu

R. Etienne-Cummings is with the department of Electrical Engineering, Johns Hopkins University. retienned jhu . edu

G. Cauwenberghs is with the division of Biological Sciences, University of California San Diego. gerte jhu . edu to the image sensor, and to transmit light to the region of interest. Fibers limit spatial resolution and force a tether on the animal. Recently, some work has been done in designing integrated image sensors [12], [13] that can be incorporated into optical systems, removing the need for optical fiber tethers.

We present the design and characterization of an optical system that could be used to optically image cortical vasculature in an awake, behaving rat without any optical or electrical tethers. We are currently working on designing CMOS image sensors that will be integrated with the system to make it fully functional. This paper describes the design and characterization of the optical system. A reference imaging system, consisting of a 12-bit cooled CCD camera (PCO, Germany) and a $60 \mathrm{~mm}$ lens (Nikon, NY), was used for testing the designed system. Cortical vasculature images acquired through the system are also presented.

\section{SYSTEM PARAMETERS}

\section{A. Illumination Parameters}

Since this optical system is designed for long term imaging from an awake and behaving animal, it is critical that the illumination level does not vary with time. Similarly, a spatial variation is also detrimental. This can introduce measurement errors into any quantitative information inferred from the images. Another important factor, given the proximity of the light source to the tissue being imaged, is a possible increase in the local temperature.

\section{B. Optical Parameters}

The simplest optical parameter of any imaging system is the magnification, the ratio of the size of the image projected to the focal plane to the actual size of the object being imaged [14]

Contrast indicates how well a system can resolve different intensities. The spatial frequency response (SFR) measures its ability to retain contrast at different spatial frequencies [14]. The SFR at low frequencies is by definition assumed unity and drops at higher frequencies, indicating the system has a limited resolution. The SFR of coupled optical systems is the product of individual SFRs.

Typically, lenses introduce distortions [14] into images. The most common of these is radial distortion. Simply put, straight lines in extrinsic space become curved lines in the camera's intrinsic (distorted) space. These distortions can be modeled as a set of linear equations [15], [16] using which it is possible to correct for the distortions. 


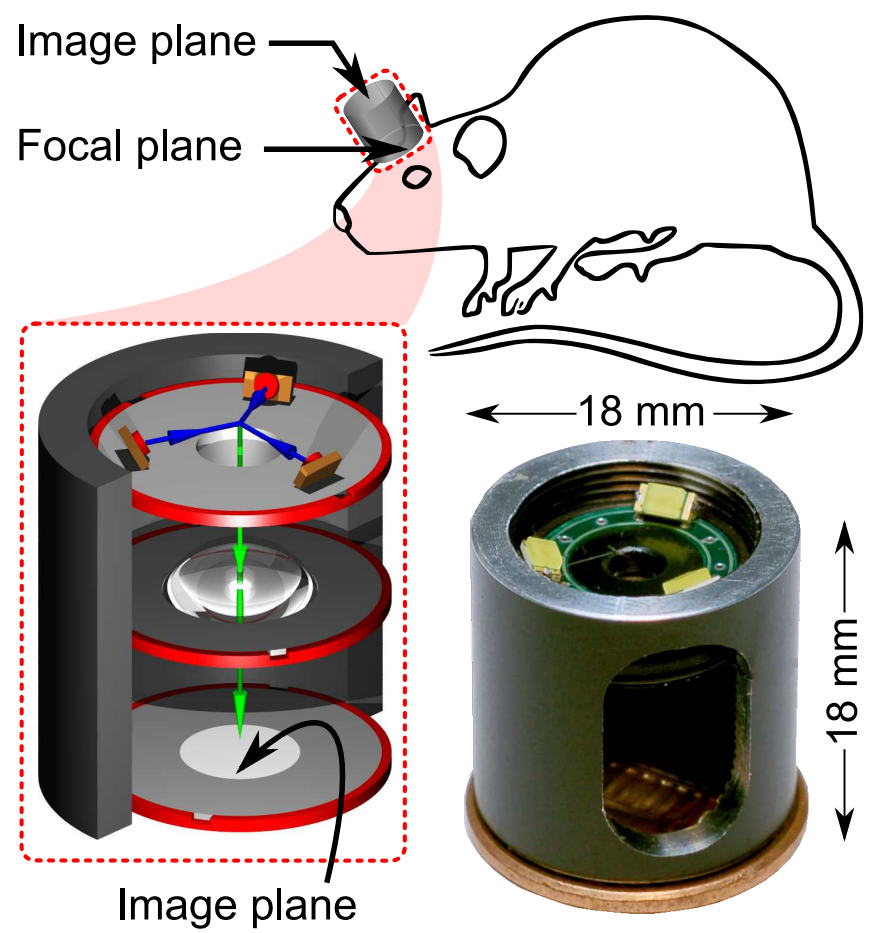

Fig. 1. Counterclockwise from top: a schematic drawing showing the eventual use of the system being described in imaging from an awake behaving animal; a schematic drawing of the optical design of the system showing the incident (blue) and reflected (green) light paths; and a photograph of the assembled system atop a US $1 \notin$ coin.

\section{SYSTEM DESIGN}

Fig. 1 shows a visualization of the system head-mounted on a rat. The figure also shows a schematic drawing of the design of the optical system illustrating the incident and reflected light paths and a photograph of the assembled system on top of a US $1 \phi$ coin. The optical housing of our system was made from a half-inch inner diameter lens tube that was internally threaded at 40 threads per inch (Thor Labs, NJ). All the elements of the system were mounted on externally threaded restraining rings (Thor Labs, NJ). Our design is based on the premise that these rings, when rotated inside the tube, will translate. The rings can be used to hold and move optical elements.

For increased efficiency and simplicity, we designed our light source to be in front of the lens rather than off the optical axis like a standard epi-fluorescent microscope, which would require a partially reflective or dichroic mirror. The light source consists of up to six surface-mount light emitting diodes (LEDs) mounted in a hexagonal fashion on a printed circuit board that is mounted on a restraining ring. LEDs offer the advantage of being spectrally narrow, highly efficient and available in miniaturized packages. The LEDs can be electronically addressed in groups of two $\left(180^{\circ}\right.$ apart), three $\left(120^{\circ}\right.$ apart $)$ or six $\left(60^{\circ}\right.$ apart $)$ allowing for multispectral imaging. However, there is a trade-off in terms of illumination uniformity as the LEDs generate a cone of light. The illumination disc can be moved away from the sample to homogenize the illumination to some extent. The remaining non-uniformity can be calibrated for in software.

To keep the design simple, we started with a single lens. Since we plan to attach our system to a rat skull, size constraints dictate a short optical path. Thus, we chose a lens with a focal length of $4.6 \mathrm{~mm}$ and numerical aperture of 0.4 (Thorlabs, NJ). Additionally, the lens was aspheric to minimize distortions. It was mounted on a custom machined piece that was press fitted into a restraining ring. Once inserted into the lens tube, the disc could be moved along the optical axis thereby permitting changes in the focal plane and the magnification. Our target magnification was around 1 , requiring the lens to be within a few millimeters of the sample. The light source described above allowed sufficient clearance for the lens to approach the sample.

\section{RESULTS}

The system described in Sec. III was assembled and a photograph is shown in Fig. 1. The complete system was $18 \mathrm{~mm}$ in height and $18 \mathrm{~mm}$ in diameter. The weight was 5.7 grams. After assembly, the ability to rotate and thereby translate all the discs was confirmed.

To characterize the performance of the optical system, we used a variety of checkerboard patterns ranging in size from 640 to $40 \mu \mathrm{m}$. Since the total optical path length of the system was small, leading to a short back focal length, we required a high-resolution image sensor which could be brought to within a few millimeters of the lens disk. We did not have access to such a sensor, so we characterized our system through a second optical system. We used a 12 bit cooled CCD camera (PCO, Germany) fitted with a $60 \mathrm{~mm}$ lens (Nikon, NY). The lens was focused at the plane where our system would project its image. The argument here is that a secondary optical system cannot improve the image quality of the front-end system [14]. The measured transfer function of the system will be the product of the transfer functions of the two subsystems. Since the best possible transfer function is unity for all spatial frequencies, the measurement will give us a lower bound on our performance. Similar arguments can be made to relate the measured magnification and distortions to those of the system under characterization.

\section{A. Illumination}

Fig. 2(a) shows the incident optical power level measured over a period of two hours using an optical power meter. The measurement was made at two different power levels. As can be seen, the power is extremely stable at the lower intensity with a peak variation of less than $0.5 \%$. At the higher intensity, the variation over two hours was about $1.1 \%$. To simulate a realistic case for measuring any local heating caused by the LEDs, the system was mounted over an agar plate and a temperature probe was inserted into the agar in the region being imaged. Fig. 2(b) plots the local temperature near the light source. Again the measurements are made at two power levels over a period of two hours. The lower and higher intensity illumination caused increases of $0.4^{\circ} \mathrm{C}$ and $0.7^{\circ} \mathrm{C}$,respectively, which are not large enough to cause discomfort or to trigger regulatory mechanisms. 

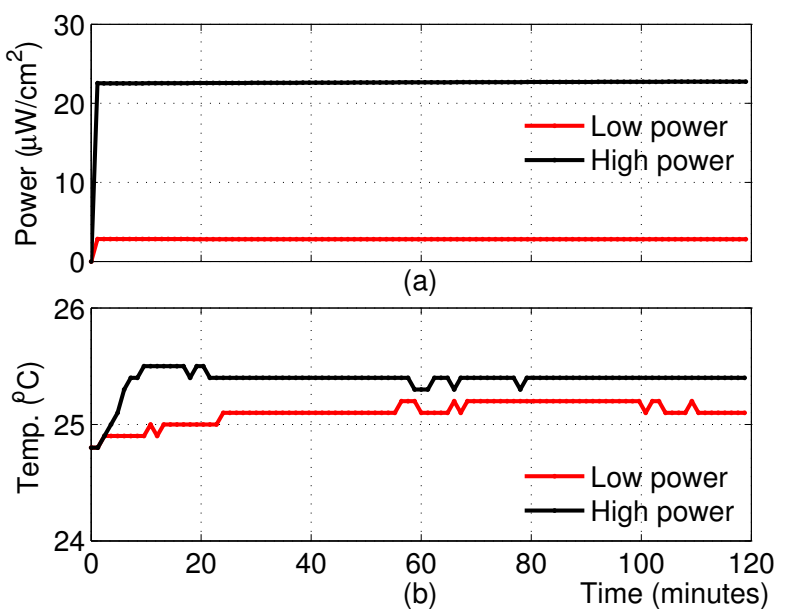

Fig. 2. (a) Incident optical power variation and (b) temperature variation in the region of interest over a period of two hours.
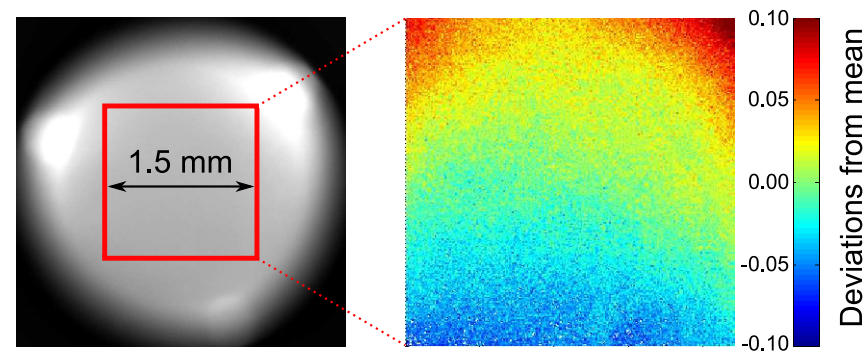

Fig. 3. Illumination non-uniformity: entire clear aperture of the system (left), deviations from the mean illumination in the region of interest (right).

To measure the spatial non-uniformity of the illumination, the optical system was mounted on a flat, featureless white surface, having previously been focused on that plane. Images were captured through the second optical system as described earlier. The exposure was adjusted such that the brightest region being imaged was just under saturation. Fig. 3 shows the entire clear aperture of the system on the left and deviations from the mean illumination over the maximum field of view of $1.5 \mathrm{~mm} \times 1.5 \mathrm{~mm}$. The standard deviation is less than $4 \%$ of the mean illumination. The image of the clear aperture shows the reason for the nonuniformity - all LEDs are not equally radiant. This leads to a smooth gradient which can be corrected for in software.

\section{B. Optics}

Fig. 4(a-c) show images of $160 \mu \mathrm{m}, 120 \mu \mathrm{m}$ and $60 \mu \mathrm{m}$ checkerboard patterns taken through our optical system. For comparison, Fig. 4(d-f) show images of the same patterns taken through the reference system alone. Qualitatively, the sharpness of the images through our system seems to be lesser than that of the reference system and distortions appear in the corners of the image. It should be noted that since the images taken by our system are actually acquired after they pass through the second system, any distortions or loss of sharpness in the reference system will also appear in our images. The absolute magnification of the images seen in

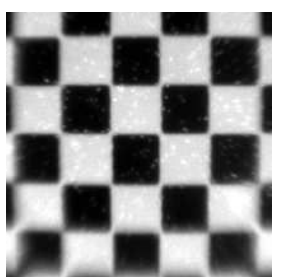

(a)

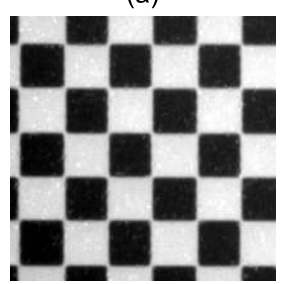

(d)

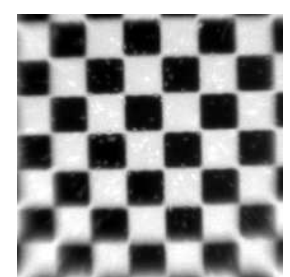

(b)

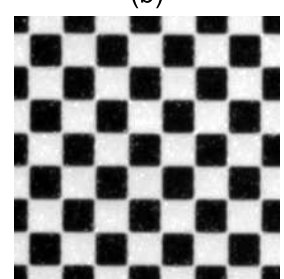

(e)

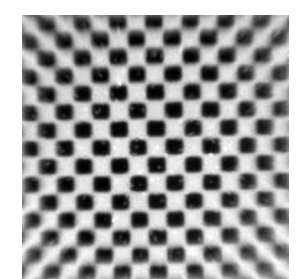

(c)

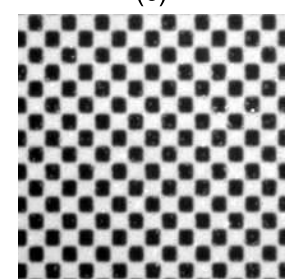

(f)
Fig. 4. Images obtained through the designed optical system (a-c) and through the reference system (d-f). (a,d), (b,e) and (c,f) checkerboard patterns of size $160 \mu \mathrm{m}, 120 \mu \mathrm{m}$ and $60 \mu \mathrm{m}$ respectively.

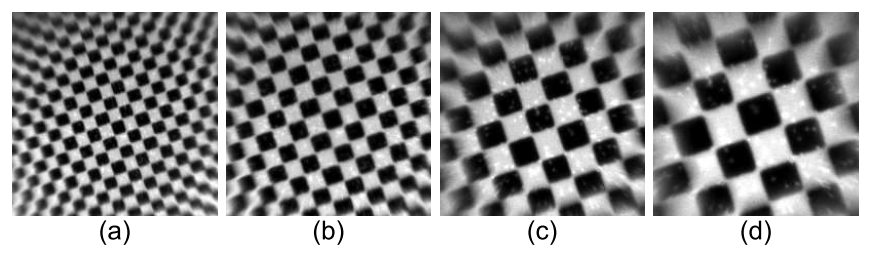

Fig. 5. Images of the $100 \mu \mathrm{m}$ checkerboard obtained through our system at different magnifications - (a) 0.8, (b) 1.1, (c) 1.7 and (d) 2.3.

Fig. 4(a-c) is 1.57 and for images in Fig. 4(d-f) is 1.37. Thus, the magnification of our system is $1.57 / 1.37=1.15$ with a field of view (FOV) of $0.9 \mathrm{~mm} \times 0.9 \mathrm{~mm}$. By translating the lens disk and the distance between the lens disk and the focal plane, we could achieve other magnifications as shown in Fig. 5.

To quantitatively compare our system to the reference system, we calculated the spatial frequency response using the slanted-edge method [17]. Fig. 6 shows this data for both the systems at a magnification of 1.15 and FOV of $0.9 \mathrm{~mm} \times 0.9 \mathrm{~mm}$. The inset in the figure shows the edges used for the calculation. The data bears out the qualitative conclusion we had drawn earlier that the reference system is slightly better than our system. It should be noted, however, that the reference system consists of a highly corrected lens $120 \mathrm{~mm}$ in length and weighing 440 grams.

We characterized optical distortions within a FOV of $1.5 \mathrm{~mm} \times 1.5 \mathrm{~mm}$ using images of the $100 \mu \mathrm{m}$ checkerboard. The results indicated no distortions at the center and a $5.3 \%$ radial distortion at the edge of the FOV which was brought down to $1.8 \%$ after correction using the Caltech calibration toolbox [18].

\section{Imaging in Animals}

The presented system lacks an integrated image sensor which prohibits us from imaging in awake animals. However, we imaged cerebral vasculature in an anesthetized rat through the system. Images were taken through the reference system 


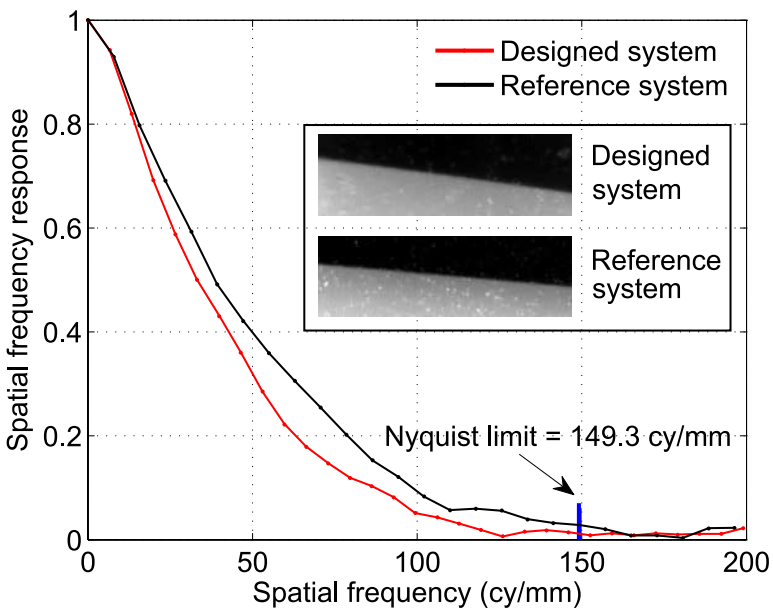

Fig. 6. Spatial Frequency Response (SFR) of the designed system compared to that of the reference system.

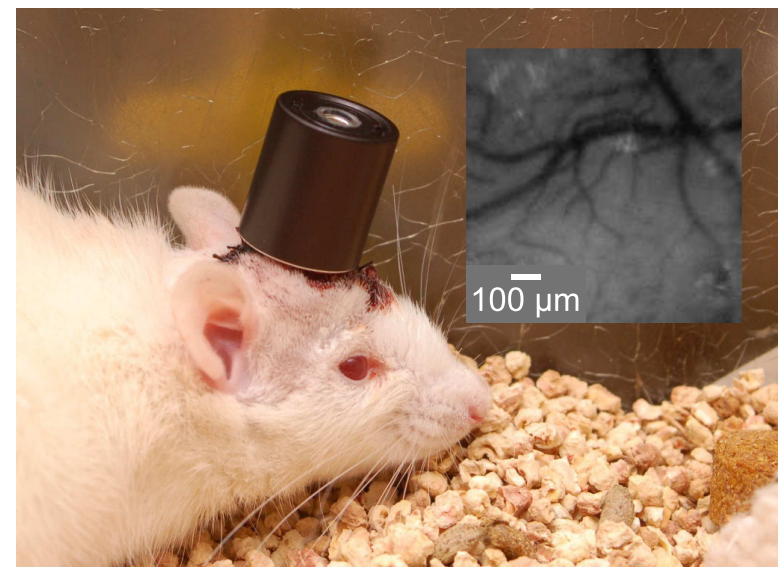

Fig. 7. Photograph showing the eventual attachment of the optical system to an awake, unrestrained rat. The inset shows an image of cortical vasculature of an anesthetized rat taken through the designed system.

described earlier. Fig. 7 shows a photograph of the eventual attachment of the designed optical system to a rat skull. While the system in the photograph is not attached to the rat, the picture conveys a sense of the scales involved. The inset in the figure shows an image of cortical vasculature seen through the system in an anesthetized rat.

\section{CONCLUSIONS AND FUTURE WORK}

We have described the design and characterization of a miniature optical imaging system. It has a small footprint of $4.6 \mathrm{~cm}^{3}$ and weighs 5.7 grams. The spatial non-uniformity of the light source was under $4 \%$ over a maximum FOV of $1.5 \mathrm{~mm} \times 1.5 \mathrm{~mm}$, while the temporal and temperature deviations were negligible. The spatial resolution and geometric distortions were comparable to a reference optical system two orders of magnitude larger. Future work involves further miniaturization, incorporation of filters for fluorescent imaging and improving the illumination uniformity.

Fig. 7 illustrates that it will be possible to mount this system directly on an animal such as a rat to perform in-vivo imaging. With that end in mind, two avenues are currently being explored. First, we are developing high sensitivity, low noise CMOS image sensors to incorporate into the system. Secondly, we are exploring ultra-wide band telemetry to allow streaming high data-rate image data for real-time analysis. We believe this work will lead to a deeper understanding of neurovascular dynamics in behavioral scenarios.

\section{ACKNOWLEDGMENTS}

The authors gratefully acknowledge the help of Jay Burns at the BME machine shop in implementing the microscope.

\section{REFERENCES}

[1] B. Winegar and M. MacIver, "Isoflurane depresses hippocampal CA1 glutamate nerve terminals without inhibiting fiber volleys," $B M C$ Neurosci., vol. 7, p. 5, 2006.

[2] J. Berg-Johnsen and I. Langmoen, "The effect of isoflurane on excitatory synaptic transmission in the rat hippocampus," Acta Anaesthesiol. Scand., vol. 36, pp. 350-355, May 1992.

[3] J. Cham, E. Branchaud, Z. Nenadic, B. Greger, R. Andersen, and J. Burdick, "Semi-Chronic Motorized Microdrive and Control Algorithm for Autonomously Isolating and Maintaining Optimal Extracellular Action Potentials," Journal of Neurophysiology, vol. 93, no. 1, pp. 570-579, 2005.

[4] B. Vos, M. Wijnants, S. Taeymans, and E. De Schutter, "Miniature carrier with six independently moveable electrodes for recording of multiple single-units in the cerebellar cortex of awake rats," Journal of Neuroscience Methods, vol. 94, no. 1, pp. 19-26, 1999.

[5] D. Rector, G. Poe, P. Redgrave, and R. Harper, "A miniature CCD video camera for high-sensitivity light measurements in freely behaving animals," J. Neurosci. Methods, vol. 78, pp. 85-91, Dec 1997.

[6] Y. Sabharwal, A. Rouse, L. Donaldson, M. Hopkins, and A. Gmitro, "Slit-scanning confocal microendoscope for high-resolution in vivo imaging," Appl. Opt., vol. 38, pp. 7133-7144, Dec 1999.

[7] C. Engelbrecht, R. Johnston, E. Seibel, and F. Helmchen, "Ultracompact fiber-optic two-photon microscope for functional fluorescence imaging in vivo," Opt. Express, vol. 16, pp. 5556-5564, Apr 2008

[8] D. Vucini and T. Sejnowski, "A compact multiphoton 3D imaging system for recording fast neuronal activity," PLoS ONE, vol. 2, p. e699, 2007.

[9] D. Dombeck, A. Khabbaz, F. Collman, T. Adelman, and D. Tank, "Imaging large-scale neural activity with cellular resolution in awake, mobile mice," Neuron, vol. 56, pp. 43-57, Oct 2007.

[10] I. Ferezou, S. Bolea, and C. Petersen, "Visualizing the cortical representation of whisker touch: voltage-sensitive dye imaging in freely moving mice," Neuron, vol. 50, pp. 617-629, May 2006.

[11] B. Flusberg, A. Nimmerjahn, E. Cocker, E. Mukamel, R. Barretto, T. Ko, L. Burns, J. Jung, and M. Schnitzer, "High-speed, miniaturized fluorescence microscopy in freely moving mice," Nature Methods, 2008.

[12] J. Ohta, A. Higuchi, A. Tagawa, K. Sasagawa, T. Tokuda, Y. Hatanaka, H. Tamura, and S. Shiosaka, "An implantable cmos image sensor for monitoring deep brain activities of a freely moving mouse," pp. 269 272, Nov. 2008

[13] J. Park, E. Culurciello, D. Kim, J. Verhagen, S. Gautam, and V. Pieribone, "Voltage sensitive dye imaging system for awake and freely moving animals," in IEEE Biomedical Circuits and Systems Conference, 2008. BioCAS 2008, pp. 89-92, 2008.

[14] E. Hecht, Optics. Addison-Wesley Pub. Co, 2001.

[15] J. Heikkila and O. Silven, "A four-step camera calibration procedure with implicit imagecorrection," in 1997 IEEE Computer Society Conference on Computer Vision and Pattern Recognition, 1997. Proceedings., pp. 1106-1112, 1997.

[16] Z. Zhang, "Flexible camera calibration by viewing a plane from unknown orientations," in Computer Vision, 1999. The Proceedings of the Seventh IEEE International Conference on, vol. 1, pp. 666-673, 1999.

[17] P. Burns, "Slanted-edge MTF for digital camera and scanner analysis," in IS \& T PICS Conference, pp. 135-138, Society for Imaging Science \& Technology, 2000.

[18] J.-Y. Bouguet, "Camera calibration toolbox for matlab," March 2009 www.vision.caltech.edu/bouguetj/calib_doc/. 